\title{
The European Society for Immunodeficiencies (ESID) Registry Working Definitions for the Clinical Diagnosis of Inborn Errors of Immunity
}

Seidel, M.G. ${ }^{1 \$ *}$, MD, Kindle, ${ }^{2}{ }^{2}, 3 \$, M D$, Dipl.Inf., Gathmann, $B .{ }^{2}, M S c$, Quinti, I. ${ }^{4}, M D$, Buckland, M. ${ }^{5}, M D$, van Montfrans, J. ${ }^{6}, M D, P h D$, Scheible, R. $^{2}$, Dipl.Inf., Rusch, S. ${ }^{2}, 3$, Dipl.Inf., Gasteiger, L.M. ${ }^{1}$, C.m., Grimbacher, B. ${ }^{2}, M D$, Mahlaoui, N. ${ }^{7 \#,}$, MD, PhD, and Ehl, S. ${ }^{2,8 \# *} M D$, on behalf of the ESID Registry Working Party (see complete contributors list at the end)

\$ contributed equally

\# jointly coordinated and supervised this work

1, Research Unit for Pediatric Hematology and Immunology, Division of Pediatric Hemato-Oncology, Department of Pediatrics and Adolescent Medicine, Medical University Graz, Graz, Austria

2, Institute for Immunodeficiency, Center for Chronic Immunodeficiency (CCI), Medical Center - University of Freiburg, Faculty of Medicine, University of Freiburg, Germany

${ }^{3}$, Central Facility Biobanking, Medical Center, Faculty of Medicine, University of Freiburg, Germany

${ }^{4}$, Dept. of Molecular Medicine, Sapienza University of Rome, Italy

${ }^{5}$, Great Ormond St Hospital for Children NHS Foundation Trust and UCL Institute of Molecular and Cellular Immunology, Institute of Child Health, London, UK.

6, Pediatric Immunology and Infectious Diseases, UMC Utrecht, Utrecht, Netherlands

7, CEREDIH, French National Reference Centre for Primary ImmunoDeficiencies and Pediatric ImmunoHematology and Rheumatology Unit, Necker-Enfants Malades University Hospital, Assistance Publique-Hôpitaux de Paris, Paris, France

8, Center for Pediatrics and Adolescent Medicine, Medical Center, Faculty of Medicine, University of Freiburg, Germany

All authors declare that they have no conflict of interest to disclose.

*Correspondence: Markus G. Seidel, M.D., Auenbruggerplatz 38, 8036 Graz, Austria, Tel.

+4331638513485; Fax. +4331638513717; email: markus.seidel@medunigraz.at; Stephan Ehl, M.D., Institute for Immunodeficiency and Children's Hospital, University Hospital Freiburg, Breisacher Straße 115, 79106 Freiburg, Tel: +4976127077300; Fax: +4976127077744, stephan.ehl@uniklinik-freiburg.de . Key Words: Primary immunodeficiency (PID); immune dysregulation (PIDD); guideline; diagnostic algorithm; classification; consensus; registry; epidemiology.

Word Count: Abstract 229; Main text: 2613; Tables: 1; Figures: 3; References: 19.

Supplementary Material: 2 Tables. 


\section{Abstract}

2 Patient registries are instrumental for clinical research in rare diseases. They help to achieve

3 a sufficient sample size for epidemiological and clinical research and to assess the feasibility

4 of clinical trials. The European Society for Immunodeficiencies (ESID) registry currently

5 comprises information on $>25,000$ patients with inborn errors of immunity (IEI). The

6 prerequisite of a patient to be included into the ESID registry is an IEI either defined by a

7 defect in a gene included in the disease classification of the international union of

8 immunological societies (IUIS), or verified by applying clinical criteria. Because a relevant

9 number of patients, including those with common variable immunodeficiency (CVID),

10 representing the largest group of patients in the registry, remains without a genetic

11 diagnosis, consensus on classification of these patients is mandatory. Here, we present

12 clinical criteria for a large number of IEI that were designed in expert panels with external

13 review. They were implemented for novel entries and verification of existing datasets from

142014 , yielding a substantial refinement. For instance, $8 \%$ of adults and $27 \%$ of children with

15 CVID (176 out of 1704 patients) were reclassified to 22 different immunodeficiencies,

16 illustrating progress in genetics, but also the previous lack of standardized disease

17 definitions. Importantly, apart from registry purposes, the clinical criteria are also helpful to

18 support treatment decisions in the absence of a genetic diagnosis or in patients with variants 19 of unknown significance. 
21

22

23

\section{Introduction}

The diagnostic evaluation for primary immunodeficiency and immune dysregulation disorders (PID or PIDD, used synonymously), currently referred to as inborn errors of immunity (IEI), is typically initiated upon the manifestation of $i$, an increased severity or frequency of infections or an infection with an opportunistic microorganism, ii, symptoms of immune dysregulation like (multi-organ or early-onset) autoimmunity or autoinflammation, and/or, iii, clinical signs of immunodeficiency in a patient with syndromic features or malignancy. Other signs like a positive family history, failure to thrive, lymphopenia, hypogammaglobulinemia, or prolonged need of intravenous antibiotic treatment are among the well-recognized alarm bells prompting physicians to initiate further testing for IEI. International consensus papers on clinical diagnostic algorithms guide the diagnostic procedure, and an increasing number of these sets of warning signs has been analyzed for sensitivity and specificity ${ }^{1-5}$.

\section{Current technologies and the delineation of the human genome have enabled next} generation sequencing diagnostics for IEI by targeted gene panels, whole exome, or genome analysis, that are becoming available in more and more countries and centers globally. Due to reduced costs as compared to historical genetic analyses and proven cost-efficiency, these novel genetic analysis tools are applied at earlier time points during hypothesis-driven diagnostic work-up ${ }^{6,7}$. Further, the inclusion of severe combined or even other profound immunodeficiencies to newborn screening programs is becoming standard in many countries around the world because these diseases fulfil the medical genetics criteria for newborn screening, and screening is cost-efficient, thereby tremendously supporting early diagnosis, improving management, and increasing survival of patients with $|\mathrm{EI}|^{8-11}$. Today, more than 340 monogenic IEI are known, and the number is increasing rapidly. The International Union of Immunological Societies (IUIS) has biennially published a classification of PIDs that classifies PIDs into 9 categories according to the underlying molecular defect ${ }^{12}$. In addition to this genetic tabular list of PID disorders with brief descriptions of main laboratory and clinical findings, recently, a phenotype-driven diagnostic consensus paper has been added to extend and improve the practical use of this classification ${ }^{13}$. The latter has also been made available as free application for mobile devices, further increasing its practical usefulness ${ }^{14,15}$. 
53

Patient registries are instrumental for clinical research in rare diseases. A registry for a large, heterogenous and phenotypically overlapping group of disorders such as IEI needs stringent criteria for disease classification to allow appropriate data entry. Ideally, the registration title (i.e., categorization) of every entry would be specific, undisputable, and verified. In the ESID registry, the registration title entry is the IEI diagnosis. The IEI diagnosis is considered definitive in cases in which a known monogenic pathological variant was identified that explains the phenotype, although functional testing of variants is not required for validation to date. However, despite the advances of genetic diagnostic technologies, there are still a majority of patients who lack a definitive genetic diagnosis. Therefore, clinical criteria were established by a panel of expert groups to correctly classify the majority of IEI disorders for patient inclusion into the ESID registry by disease category even if a genetic cause is unknown.

The ESID online registry was founded in 2004 and fulfils the role of a central IEI patient registry in Europe and some countries from other continents. It is a platform for clinical trials and other research projects. It also represents a growing network of centers, connecting experts, immunological societies, and other stakeholders. This important role of the registry underpins the relevance of a stringent and reliable data set quality, setting the ground for quality studies in our field. Examples of published and ongoing studies using the ESID registry data are the Activated PI3-Kinase Delta Syndrome (APDS) study, the study on unclassified predominantly antibody deficiencies (UnPAD) study, the Common variable immunodeficiency (CVID) burden study, or a study on patients with Ataxia teleangiectasia ${ }^{16}$, ${ }^{17}$. Numerous further papers using or highlighting the ESID registry have been published; please refer to the ESID registry publications website for an overview ${ }^{18}$.

A substantial amount of ESID registry data can be accessed by the public at the ESID registry web page ${ }^{19}$, whereas more specific and detailed data can be retrieved and analyzed only by ESID registry members of a documenting center upon login. Thirdly, data usage by third parties may be requested by submitting a research project proposal to the ESID registry working party or may be negotiated and is subject to a contract between the ESID and the institution/party requiring access. Data from the United Kingdom Primary Immunodeficiency 
85

Network (UKPID) are imported on a weekly interval, so that the total amount of data computed by the reporting/analysis tool are updated weekly. Publicly available ESID registry reports include: number of patients in the registry, distribution between children and adults for every country, ESID registry patient numbers and proportions per IEI main diagnosis category and per country, yielding a map of the minimal prevalence of IEI, ESID registry data on hematopoietic stem cell transplantation and gene therapy ${ }^{19}$. The "members only" section allows more specific analyses for the patients entered by the member's documenting center and the total of patients in the registry: e.g., to show and export a list of IEI categories, subcategories, specific IEI diagnoses, and gene defects, to retrieve information on the country and sex distribution as well as the rate of coverage (difference from the expected geographical prevalence).

When the ESID registry was created, no central disease classification manual was available. The registry was then entirely restructured for quality assurance and data utility purposes in 2014. During the data transfer process from the previous to the current version, an obligatory verification step of the main title of an existing or of a novel entry, i.e., the IEI diagnosis, was implemented. Thus, upon choosing a diagnosis, the online entry system automatically generates a query asking whether the defined clinical criteria for the chosen diagnosis are fulfilled. The data manual also proposes to consider a number of alternative classifications if the criteria are not completely fulfilled. The present catalogue of phenotypical criteria was designed to enable correct disease classification for patients with IEI who lack a definite genetic diagnosis at the time of registry inclusion, and, similar to the IUIS documents described above, represents continually updated work in progress. 


\section{Materials and Methods}

110 For each of 92 clinical IEI entities to be verified or excluded in patients who lack a genetic

111 diagnosis, a number of mandatory and suggestive clinical features was defined by

112 international experts and collected between 2013 and 2018. Drafts of proposed criteria

113 were elaborated by experts in the field and were subsequently peer reviewed by one or

114 more external experts in the respective category of IEI before implementation. Contributors

115 and reviewers of each entity are stated. A regular quality check and update of these criteria

116 at a biennial basis is being coordinated through the ESID registry working party chair. For the

117 illustration of diagnosis transition after implementation of the diagnosis verification process,

118 we analyzed the reclassification of entries of common variable immunodeficiency (CVID;

$119 n=1704$ ) upon, $i$, clinical criteria, or, $i i$, results of genetic testing in children and adults by

120 drawing a Sankey diagram (The Sankey Diagram Generator, Acquire Procurement Services,

121 Brisbane, Queensland, Australia; http://sankey-diagram-generator.acquireprocure.com/).

122 
124 Results: Clinical Diagnosis Criteria for IEl and their Application

125 The document titled ESID Registry - Working Definitions for Clinical Diagnosis of IEI is

126 available in the Online Repository of this article (see Supplementary Table 1 in the Online

127 Repository) and, in a regularly updated version, on the ESID website ${ }^{20}$. Recently, each

128 diagnosis of the compilation was supplemented with OMIM (Online Mendelian inheritance in

129 Man) numbers of corresponding, genetically defined, diagnosis entities if available, and the

130 respective category (1-9) of IEI according to the IUIS classification. This catalog may be

131 downloaded and used for individual verification of a suspected IEI diagnosis before inclusion

132 into the ESID registry. Further, upon initiation of a novel entry with a certain registration title

133 (i.e., IEI diagnosis), a pop-up window showing the respective criteria opens and requires

134 their confirmation. Figure 1 illustrates the simple steps of including a patient into the ESID

135 registry and verifying her/his diagnosis.

136

137 To analyze the benefit and demonstrate the effect of the implementation of a mandatory

138 verification process, we evaluated the records of CVID in children ( $<18$ years of age) and in

139 adults before and after application of the diagnostic criteria in 2014. The clinical diagnostic

140 criteria of CVID and, for comparison, of Unclassified antibody deficiency, and of Combined

141 immunodeficiency (CID) are shown in Table 1. Of 1704 patients with the original diagnosis of

142 CVID who were present in the registry when the verification process was implemented, 176

143 (10.3\%) were reclassified into different diagnoses. Twenty-four were reclassified on the basis

144 of a detected monogenic defect not listed under CVID (13.6\%), and 152 (86.4\%) because

145 they did not fulfill the consensus clinical CVID criteria (Figure 2, and Supplementary Table 2

146 in the Online Repository). Vice versa, 62 patients with other humoral immunodeficiencies

147 (i.e., Other hypogammaglobulinemia, Isolated IgG subclass deficiency,

148 Agammaglobulinemia, or Other humoral or unclassified immunodeficiency) were reclassified

149 to CVID during the verification process (Figure 2). Those who changed from CVID to other

150 diagnoses based on mere clinical criteria were redefined as Unclassified antibody deficiency

151 ( $n=90 ; 51.1 \%)$, Isolated IgG subclass deficiency $(n=15 ; 8.5 \%)$, Unclassified immunodeficiency

152 ( $n=10 ; 5.7 \%)$, Combined immunodeficiency $(n=10 ; 5.7 \%)$, Agammaglobulinemia $(n=3 ; 1.7 \%)$,

153 or other, rare, immunodeficiencies ( $n=24 ; 13.6 \%$; Figure 2; see also Supplementary Table 2

154 in the Online Repository for more details). Patients originally classified as CVID who were

155 reclassified to another diagnosis upon detection of a known genetic mutation were, in total, 
15624 (13.6\%), and comprised various combined immunodeficiencies ( $n=13 ; 7.4 \%)$,

157 Agammaglobulinemia $(n=5 ; 2.8 \%)$, or various other genetic diagnoses $(n=6 ; 3.4 \%)$ (Figures 2

158 and 3; and Supplementary Table 2 in the Online Repository). For a comparison of the

159 changes in diagnosis between children and adults we performed this analysis separately,

160 showing that a substantially larger proportion of children than of adults previously entered

161 under CVID changed their diagnosis (27.3\% vs. 7.7\%). Interestingly, the proportion of genetic

162 versus clinical redefinition during the routine diagnosis verification process was double in

163 adults (19 of 114 adult patients, $16.6 \%$ genetic redefinition) as compared to children (5 out

164 of 62 children, $8.1 \%$ genetic redefinition). However, the final distribution of diagnostic

165 entities after reclassification was similar between children and adults (Figure 3; and

166 Supplementary Table 2 in the Online Repository). 
168

169

170

171

172

173

174

175

176

177

178

179

180

181

182

183

184

185

186

187

188

189

190

191

192

193

194

195

196

197

198

199

\section{Discussion}

The present document describes the development and current version of the ESID Registry Working definitions for clinical diagnosis of PID/IEI as of December 2018, and comprises the entire spectrum of primary immunodeficiencies covered by the ESID registry to date. As it uses clinical disease definitions rather than separate genetic defects, this list may appear shorter than those provided in the IUIS documents. The document was designed to enable correct classification of patients without known genetic cause of their disease within the ESID registry both for novel patient inclusions and for a mandatory verification process of existing entries starting from 2014. Furthermore, these "ESID registry Clinical diagnosis criteria" are useful in clinical practice when making a working diagnosis of IEI in a patient who either lacks a genetic diagnosis or has a variant of unknown significance.

To demonstrate the effect of the introduction of a mandatory verification process of a clinical diagnosis entered into the ESID database, we chose CVID as an example, because of its high frequency among entries in the ESID registry (to date, 4,773 of 25,023 patients [19\%]) and its large proportion of patients lacking a defined genetic defect $(4,593$ of 4,773 [96\%] were merely clinically defined). The reclassification of a substantial proportion of patients with CVID, namely $27.3 \%$ of children and adolescents, and $7.7 \%$ of adults formerly entered under CVID into 22 other diagnoses reflects that a much higher resolution of the main item, i.e., the IEI diagnosis, was achieved by implementing this obligatory step (Figure

1, step 2). Previously, patient classification solely depended on the assessment and choice of the physician or documentarist who entered the patient. The biggest target group of patients who changed their diagnosis from CVID to another were those later listed under Unclassified antibody deficiencies, probably due to the fact that the criteria of the latter entity practically represent a subset but not all of those needed for CVID (Table 1). That more than 1 out of 4 children originally entered under CVID were reclassified indicates that the diagnosis of CVID is still being used too often in children, and, is important insofar as the identification of other diagnoses such as CID might imply a completely different therapeutic concept, e.g., stem cell transplantation or targeted treatment. These observations suggest the requirement of a consensus definition of CVID in childhood, for which the present criteria might be a valid backbone. 
200

201

202

203

204

205

206

207

208

209

210

211

212

213

214

215

216

217

218

219

220

221

222

223

224

225

226

227

228

229

230

231

That a large proportion of patients who were later classified as Combined immunodeficiency or as Agammaglobulinemia instead of CVID is due to the identification of a genetic cause is no surprise. However, it is interesting that a much larger proportion of adults than of children with CVID underwent successful genetic diagnostics and were reclassified. However, because the ESID registry did not record negative genetic testing for patients classified and registered before verification, it is not possible to distinguish whether this difference is due to a higher proportion of adult patients as compared to children with a clinical phenotype of CVID who underwent successful genetic testing, or whether a larger proportion of children had already undergone genetic testing prior to classification and had been classified as monogenic IEI other than CVID. Likely, this difference will disappear with increased application of next generation sequencing panel, exome, or genome diagnostics in all age groups driven by the availability of targeted treatment approaches.

An increasing number of patients with clinical features of IEI undergoes next generation sequencing diagnostics, but detected variants do not always represent variants known to explain the respective disease phenotype. The latest catalog of genes known to be potentially mutated in IEI and available for selection in the ESID registry for a patient entry, termed "ESID Online Registry - List of Diseases and Genes" can be downloaded from the ESID website ${ }^{21}$. If a known genotype can be associated with multiple phenotypes and is thus listed under various disease entities, as, for instance, the case in a RAG1 mutation, then the clinical diagnosis as defined by the documenting physician is required for the selection of the patient's registration title, i.e. the IEI diagnosis (e.g., SCID, Omenn syndrome, atypical SCID, etc.), but the application of clinical criteria is not needed. Until now, the ESID registry data section on genetic information does not collect information on variants of unknown significance (VUS), heterozygous variants that may be disease-causing, copy number variations, and it does not capture digenic or polygenic effects except for a free text entry possibility for "additional genes". Further, with the only exception of STAT3, the differences between gain- or loss-of-function mutations, dominant negative effects, or haploinsufficiency are not distinguished. In the light of the challenges and needs arising from next generation sequencing, a future version of the registry tab on genetic data should ideally collect information on the exact position of a mutation, the possibility of multiple gene defects, likely pathogenic variants, the functional effect of a detected mutation (if 
232 known or tested, and how), VUS, and combine them with more refined phenotypic details.

233 Undoubtedly, these additions will require a substantial amount of programming work and

234 resources, increasing the cost of information technology and maintenance on one hand, and

235 more time per patient and dedication to accuracy of the documentarist, bearing the risk of a

236 decrease in data completeness, quality, and stringency on the other hand.

238 Additionally, in a subgroup of patients in whom a known genetic underpinning of IEI is 239 identified, the phenotype differs from the expected, genotype-associated, clinical picture.

240 Some of these patients might have dual or multiple genotypes, leading to a mixed

241 phenotype. In another subgroup of patients the disease course might be progressive, leading

242 to a shift from one, e.g., CVID to CID or another IEl category. Today, unfortunately, such

243 genotypical or phenotypical variations that represent potentially valuable additions to

244 previous knowledge are not recorded within the ESID registry. If a patients' phenotype

245 changes from one IEI diagnosis to another, and the gene defect is also listed under the new

246 category, he can be reclassified to the new diagnosis. This new PID-diagnosis and the

247 complete history of previous documented diagnoses is recorded and shown in the user

248 interface. Further shortcomings are, e.g., that the system does not supervise the registration

249 of patients with mutations that are not disease-causing, which is left to the interpretation of

250 the documenting person; and, the current system fails to account for patients who present

251 with atypical phenotypes, if no disease-causing mutation has been identified. For now, the

252 prime requisite for inclusion of a new patient into the ESID registry is the correct definition

253 of an IEI diagnosis and its confirmation by the documentarist or physician. Currently, this

254 step is not monitored or curated on a general basis. However, in specific sub-projects (see

255 level 2 and level 3, below), data monitoring is the responsibility of the respective study

256 project committee and might be carried out for quality assurance on a study-specific basis.

257 In its current form, the first level of an entry in the ESID registry with a defined IEI

258 registration title (e.g., "CVID") does not collect a vast number of additional patient- and

259 disease-specific items other than type of presenting symptom (e.g., infection, immune

260 dysregulation, syndromic features, malignancy), diagnostic delay, way to and method of

261 diagnosis, and main treatment modality (e.g., immunoglobulin replacement, stem cell

262 transplantation, gene therapy), because experience has shown a tendency that the quality of

263 data sets decreases with increasing size. However, optional additional levels of entries (level 
2642 and level 3 studies for subsets of patients) were created within the ESID registry for the 265 purpose of answering hypothesis-driven study questions. The present Working definitions 266 for clinical diagnosis of PID/IEI provide the function of a standardized phenotypic diagnostic 267 classification process and thereby enhance the discriminative depth and quality of individual datasets within the ESID registry without burdening participants with additional need to describe features that underlie the diagnosis after patient inclusion. In future, it may be conceivable to record the confirmatory steps of clinical criteria when they are applied during patient inclusion, for instance by recording "clicks" and translating this information into a standardized clinical code terminology, to accumulate even more individual disease-specific information. In line, the implementation of a yearly phenotype follow-up questionnaire, based on the same disease-specific clinical diagnostic criteria as at inclusion, might allow the collection of new important data on the natural disease courses, e.g., in entities with progressive disease phenotypes, and to relate that to genetic data in future.

The usefulness and quality of data extracted from patient registries for rare diseases largely depends on correct data entry. It is thus of utmost importance for the ESID registry's quality assurance to review and check the disease classification of any newly added patient. With implementation of clinical criteria for 92 entities of IEI for patients who lack a monogenic underpinning of their disease, a substantial gain in refinement of the ESID registry disease cohorts was achieved as demonstrated for CVID. Moreover, apart from their use for correct classification in the ESID database, we deem these criteria highly useful for making the correct diagnosis of IEI in the clinical setting. They may also be used to guide clinical and laboratory investigations, and support or dispute IEI working diagnoses that are not genetically confirmed. An extension of the use of these comprehensive, stringent, and consensus definitions of IEI for additional purposes such as clinical studies (e.g., as inclusion or exclusion criteria), for establishing an IEI diagnosis, and for teaching purposes in clinical immunology is warranted. Together, the ESID registry clinical diagnostic criteria set a standard for making a diagnosis in IEI, either in patients without genetic diagnosis, as a starting point to make a genetic diagnosis, or in support of a definitive genetic diagnosis. 


\section{Acknowledgements}

The authors would like to thank PPTA (https://www.pptaglobal.org) and its member companies for financial support of the ESID registry. MGS is in part funded by the Styrian Children's Cancer Aid (Steirische Kinderkrebshilfe) Foundation. SE acknowledges support by the BMBF (01EO1303) and the DFG (Eh145/9-1 EURO-CID). GK, RS, and SR acknowledge support by the BMBF (BMBF 01GM0896, 01GM1111B, 01GM1517C and 01EO1303).

\section{Conflict of interest statement}

The authors declare no conflict of interests.

\section{Author contributions}

SE, NM, BGrimbacher, MB, IQ, JVM, and MGS contributed data sets, added and edited parts of the manuscript text; SE and NM jointly coordinated the work; GK, BGathmann, SR, and RS collected and analyzed ESID registry data and clinical criteria; LMG helped with the content and structure of the tables; MGS wrote the first draft of the paper and designed the figures.

\section{Contributor list (excluding those already listed as coauthors on the title page; in} alphabetical order)

Mario Abinun, Michael Albert, Sarah Beaussant Cohen, Jacinta Bustamante, Andrew Cant, Jean-Laurent Casanova, Helen Chapel, Genevieve de Saint Basile, Esther de Vries, Inderjeet Dokal, Jean Donadieu, Anne Durandy, David Edgar, Teresa Espanol, Amos Etzioni, Alain Fischer, Bobby Gaspar, Richard Gatti, Andrew Gennery, Sofia Grigoriadou, Steven Holland, Gritta Janka, Maria Kanariou, Christoph Klein, Helen Lachmann, Desa Lilic, Ania Manson, Natalia Martinez, Isabelle Meyts, Nicolette Moes, Despina Moshous, Benedicte Neven, Hans Ochs, Capucine Picard, Ellen Renner, Frederic Rieux-Laucat, Reinhard Seger, Annarosa Soresina, Dominique Stoppa-Lyonnet, Vojtech Thon, Adrian Thrasher, Frank van de Veerdonk, Anna Villa, Corry Weemaes, Klaus Warnatz, Beata Wolska, and Shen-Yin Zhang. 
1. Modell V, Quinn J, Ginsberg G, Gladue R, Orange J, Modell F. Modeling strategy to identify patients with primary immunodeficiency utilizing risk management and outcome measurement. Immunol Res 2017; 65:713-20.

2. Lankisch P, Schiffner J, Ghosh S, Babor F, Borkhardt A, Laws HJ. The Duesseldorf warning signs for primary immunodeficiency: is it time to change the rules? J Clin Immunol 2015; 35:273-9.

3. Farmand $S$, Baumann $U$, von Bernuth $H$, Borte $M$, Foerster-Waldl E, Franke $K$, et al. [Interdisciplinary AWMF guideline for the diagnostics of primary immunodeficiency]. Klin Padiatr 2011; 223:378-85.

4. Arkwright PD, Gennery AR. Ten warning signs of primary immunodeficiency: a new paradigm is needed for the 21st century. Ann N Y Acad Sci 2011; 1238:7-14.

5. Costa-Carvalho BT, Grumach AS, Franco JL, Espinosa-Rosales FJ, Leiva LE, King A, et al. Attending to warning signs of primary immunodeficiency diseases across the range of clinical practice. J Clin Immunol 2014; 34:10-22.

6. Heimall JR, Hagin D, Hajjar J, Henrickson SE, Hernandez-Trujillo HS, Tan Y, et al. Use of Genetic Testing for Primary Immunodeficiency Patients. J Clin Immunol 2018; 38:3209.

7. Meyts I, Bosch B, Bolze A, Boisson B, Itan Y, Belkadi A, et al. Exome and genome sequencing for inborn errors of immunity. J Allergy Clin Immunol 2016; 138:957-69.

8. King JR, Hammarstrom L. Newborn Screening for Primary Immunodeficiency Diseases: History, Current and Future Practice. J Clin Immunol 2018; 38:56-66.

9. Kwan A, Puck JM. History and current status of newborn screening for severe combined immunodeficiency. Semin Perinatol 2015; 39:194-205.

10. van der Spek J, Groenwold RH, van der Burg M, van Montfrans JM. TREC Based Newborn Screening for Severe Combined Immunodeficiency Disease: A Systematic Review. J Clin Immunol 2015; 35:416-30.

11. Modell V, Knaus M, Modell F. An analysis and decision tool to measure cost benefit of newborn screening for severe combined immunodeficiency (SCID) and related Tcell lymphopenia. Immunol Res 2014; 60:145-52.

12. Picard C, Bobby Gaspar H, Al-Herz W, Bousfiha A, Casanova JL, Chatila T, et al. International Union of Immunological Societies: 2017 Primary Immunodeficiency Diseases Committee Report on Inborn Errors of Immunity. J Clin Immunol 2018; 38:96-128.

13. Bousfiha A, Jeddane L, Picard C, Ailal F, Bobby Gaspar H, Al-Herz W, et al. The 2017 IUIS Phenotypic Classification for Primary Immunodeficiencies. J Clin Immunol 2018; 38:129-43.

14. Phenotypical classification of PIDD. 2017. [Android]. 2017.] Available from https://play.google.com/store/apps/details?id=com.horiyasoft.pidclassification.

15. Phenotypical classification of PIDD, iTunes. 2017. 2017.] Available from https://itunes.apple.com/us/app/pid-phenotypical-diagnosis/id1160729399?mt=8.

16. Maccari ME, Abolhassani H, Aghamohammadi A, Aiuti A, Aleinikova O, Bangs C, et al. Disease Evolution and Response to Rapamycin in Activated Phosphoinositide 3Kinase delta Syndrome: The European Society for Immunodeficiencies-Activated Phosphoinositide 3-Kinase delta Syndrome Registry. Front Immunol 2018; 9:543. 
370 17. Registry Working Party Studies. 2018.] Available from https://esid.org/WorkingParties/Registry-Working-Party/Studies.

18. ESID Registry Publications. 2018.] Available from https://esid.org/Working-

19. $\frac{\text { Parties/Registry-Working-Party/Registry-publications. }}{\text { ESID Registry Reporting. 2019.] Available from https://esid.org/Working- }}$

Parties/Registry-Working-Party/Reporting-website.

20. Primary Immunodeficiency Diagnosis Criteria. 2018.] Available from

https://esid.org/Working-Parties/Registry-Working-Party/Diagnosis-criteria.

21. ESID Registry List of Diseases and Genes. 2019.] Available from

381 https://esid.org/Working-Parties/Registry-Working-Party/ESID-Registry/List-ofdiseases-and-genes. 
382

383

384

385

386

387

388

389

390

391

392

393

394

395

396

397

398

399

400

401

402

403

404

405

406

407

408

409

\section{Legends}

Table 1. Examples of the ESID Registry - Working Definitions for Clinical Diagnosis of PID for Common variable immunodeficiency (CVID), Unclassified (predominantly) antibody deficiencies, and Combined immunodeficiencies (CID). PID, primary immunodeficiency.

Figure 1. Simplified algorithm of a patient entry or diagnosis verification process in the ESID registry. ESID, European Society for Immunodeficiencies; IEI, inborn errors of immunity.

Figure 2. The ESID registry entries under the diagnosis of common variable immunodeficiency (CVID) before (left, $n=1704$ ) and after (right, $n=1590$ ) obligatory application of the ESID clinical criteria OR entry of a genetically confirmed diagnosis (direction from left to right). Other humoral immunodeficiencies that were later classified as CVID are shown in yellow (total $n=62$ ); entries with CVID that were confirmed as CVID $(n=1528)$ or reclassified under a different IEI category based on clinical criteria are marked in green ( $n=152 ; 86.4 \%$ of reclassified patients from CVID) or, if based on genetic criteria, in purple ( $n=24 ; 13.6 \%)$, and are grouped for clarity. The thickness of lines/bars corresponds to the relative patient number. More detailed data are shown in Supplementary Table 2 in the Online Repository.

Figure 3. The subgroup of patients previously entered under CVID who were reclassified ( $n=176)$ is shown separately for adults (blue) and children (red), and represents the bottom $10.3 \%$ of the dark grey bar on the left panel of Figure 2. Reclassification from CVID on the left was undertaken by using clinical diagnostic criteria (green) or a genetic diagnosis (purple) on the right, distinguishing children (red) and adults (blue) out of the total of 1704 patients with the diagnosis of CVID (1477 adults and 227 children, of whom 1363 and 165, respectively, were verified as CVID and are shown in Figure 2). The thickness of lines/bars corresponds to the relative patient number. More detailed data are shown in Supplementary Table 2 in the Online Repository. 
Table 1. Examples of the ESID Registry - Working Definitions for Clinical Diagnosis of PID for Common variable immunodeficiency (CVID), Unclassified (predominantly) antibody deficiencies, and Combined immunodeficiency (CID).

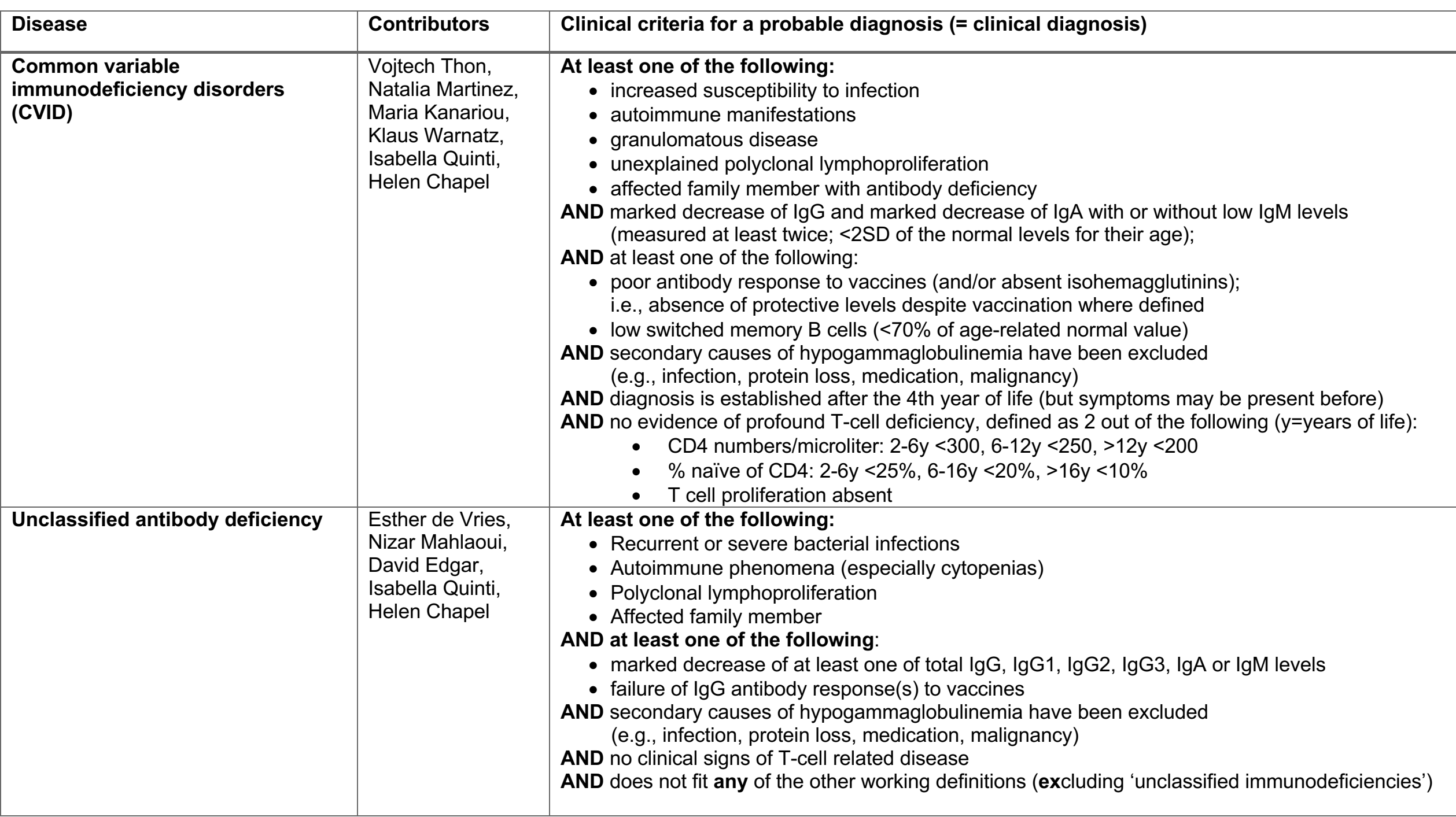




\begin{tabular}{|c|c|c|}
\hline Disease & Contributors & Clinical criteria for a probable diagnosis (= clinical diagnosis) \\
\hline Combined immunodeficiency (CID) & $\begin{array}{l}\text { Stephan Ehl, } \\
\text { Maria Kanariou, } \\
\text { Alain Fischer }\end{array}$ & $\begin{array}{l}\text { At least one of: } \\
\text { - at least one severe infection (requiring hospitalization) } \\
\text { - one manifestation of immune dysregulation } \\
\text { (autoimmunity, IBD, severe eczema, lymphoproliferation, granuloma) } \\
\text { - malignancy } \\
\text { - affected family member } \\
\text { AND } 2 \text { of } 4 \text { T cell criteria fulfilled: } \\
\text { - reduced CD3 or CD4 or CD8 T cells (using age-related reference values) } \\
\text { - reduced naïve CD4 and/or CD8 T cells } \\
\text { - elevated g/d T cells } \\
\text { - reduced proliferation to mitogen or TCR stimulation } \\
\text { AND HIV excluded } \\
\text { AND exclusion of a clinical diagnosis associated with CID } \\
\text { (e.g., defined syndromic diseases, DKC, AT, CHH) }\end{array}$ \\
\hline
\end{tabular}

